\title{
Viewpoint \\ ADAM metalloproteases and EGFR signalling
}

Julia MW Gee and Janice M Knowlden

Tenovus Centre for Cancer Research, Welsh School of Pharmacy, Cardiff University, Wales, UK

Corresponding author: Julia MW Gee (e-mail: gee@cardiff.ac.uk)

Published: 21 July 2003

Breast Cancer Res 2003, 5:223-224 (DOI 10.1186/bcr637)

(C) 2003 BioMed Central Ltd (Print ISSN 1465-5411; Online ISSN 1465-542X)

\section{Introduction}

Epidermal growth factor receptor (EGFR) comprises a dominant signalling pathway for many tumours. In breast cancer, increased expression of EGFR or its EGF-like ligands and hyperactivation of subsequent mitogen-activated protein kinase (MAPK) and protein kinase B (AKT) signalling have been linked to anti-hormone resistant growth, metastatic progression and poor prognosis. Considerable data exist detailing EGFR activation and the downstream signalling that is triggered subsequent to EGFR ligand binding, allowing rationale design of several anti-EGFR agents that are currently in clinical trials in cancers including the breast. However, less is known about the mechanisms regulating EGF-like ligand availability and their potential as therapeutic targets.

EGFR ligands, including transforming growth factor $\alpha$ (TGF $\alpha$ ), heparin-binding EGF (HB-EGF) and amphiregulin (AR), are expressed as transmembrane precursors. These are released from the cell surface following shedding of the extracellular domain (ectodomain shedding) by zincdependent proteinases. The soluble ligand can bind and activate EGFR in an autocrine or paracrine manner, while the transmembrane (pro) form may activate EGFR in adjacent cells (juxtacrine). Of emerging importance as regulators of EGFR ligand shedding is the ADAM (a disintegrin and metalloprotease) family of membrane glycoproteins. This family currently comprises 34 members. However, the nature of individual ADAMs required for EGFR ligand shedding, and their role in EGFR activation and its interaction with other signalling pathways, remain poorly defined in tumour cells. Three new articles examining ADAM17 (TNF $\alpha$-converting enzyme; TACE) and ADAM10 (Kuzbanian) shed light in these areas. Borrell-Pages et al. [1] establish relevance of TACE to TGF $\alpha$ shedding and EGFR signalling in clinical breast cancer, while Gschwind et al. [2] and Yan et al. [3] reveal that TACE and ADAM10 contribute to the interplay between EGFR and diverse signalling pathways.

\section{ADAM metalloprotease TACE controls TGF $\alpha$ shedding in tumours in vivo and associates with EGFR activation in breast cancer}

The new article by Borrell-Pages et al. provides compelling evidence that EGFR activation by TGF $\alpha$ is critically dependent on ligand shedding in tumours. Interestingly, shedding not only controls availability of the soluble ligand but also determines juxtacrine activity of the transmembrane form. The ADAM TACE was revealed to be the key regulator for TGF $\alpha$ shedding in transfected Chinese hamster ovary $(\mathrm{CHO})$ cells. These data complement previous in vitro observations that absence of/non-functional TACE underlies defective TGF $\alpha$ shedding. Excitingly, Borrell-Pages et al. demonstrated a crucial role for TACE in tumourigenesis, with TACE-mediated TGF $\alpha$ shedding being essential for EGFR activation and maximal tumour development of the transfected $\mathrm{CHO}$ cells in vivo. Furthermore, TACE was markedly overexpressed in human breast cancer, where it directly associated with EGFR activation. TACE thus appears to be of considerable importance in regulating EGFR signalling in breast tumours. The prognostic implications of heterogeneity of TACE expression remain to be addressed. Similarly, monitoring of TACE in endocrine resistant breast cancer and clinical progression is required, particularly since metalloprotease-mediated EGFR ligand shedding regulates breast cancer cell proliferation and migration in vitro [4].

\section{ADAMs contribute to EGFR transactivation by diverse signalling pathways}

Multiple pathways can promote EGFR signalling and growth by EGFR transactivation. EGFR is thus an important conduit for relaying multiple signalling inputs. Many "cross-talk" mechanisms have been described, including

$\mathrm{ADAM}=$ a disintegrin and metalloprotease; $\mathrm{AKT}=$ protein kinase $\mathrm{B} ; \mathrm{AR}=$ amphiregulin; $\mathrm{EGF}=$ epidermal growth factor; $E \mathrm{EFR}=$ epidermal growth factor receptor; GPCR $=\mathrm{G}$ protein coupled receptor; HB-EGF = heparin-binding epidermal growth factor; IGFR $=$ insulin-like growth factor receptor; $\mathrm{MAPK}=$ mitogen-activated protein kinase; MMP $=$ matrix metallo protease; TACE $=$ tumour necrosis factor $\alpha$-converting enzyme; TGF $\alpha=$ transforming growth factor $\alpha$. 
those employing metalloprotease-mediated shedding of EGFR ligands. G protein-coupled receptors (GPCR) transactivate EGFR in diverse cells, an interplay previously thought to be largely EGFR ligand-independent. However, building on observations that GPCR can also transactivate EGFR via metalloprotease induction of EGF-like ligands in several tumour cell types, Yan et al. have discovered that ADAM10 promotes HB-EGF release in COS-7 and PC3 cancer cells. In parallel, Gschwind et al. have demonstrated in head and neck cancer cells that GPCR transactivation of EGFR is regulated by TACE cleavage of $A R$, resulting in MAP kinase and AKT signalling, proliferation and cell migration. Interestingly, metalloproteasemediated GPCR-EGFR cross-talk has also been reported in breast cancer [5]. Thus, oestrogens rapidly transactivate EGFR to hyperactivate MAP kinase via G protein coupled receptor (GPR30)-dependent promotion of HBEGF at the plasma membrane, an event that occurs in oestrogen receptor positive and negative breast cancer cells [5]. Of some further interest is insulin-like growth factor receptor (IGFR), which induces EGFR activation via metalloprotease-dependent release of AR or HB-EGF in several tumour cell types [6]. IGFR-EGFR cross-talk is certainly apparent in mammary epithelial cells, since EGFR blockade abrogates IGFR-driven MAP kinase signalling and primes apoptosis. However, it remains controversial whether this cross-talk involves metalloprotease-mediated EGFR ligand release [7] and studies investigating IGFREGFR interactions are ongoing in breast cancer. While the matrix metalloproteases MMP2 and MMP9 appear to play a role in GPCR-mediated HB-EGF release, the full profile of metalloproteases, including ADAMs, contributing to EGFR transactivation and its end-points remains to be defined in breast cancer.

\section{Conclusion}

Collectively the recent articles by Borrell-Pages et al, Yan et al, and Gschwind et al. demonstrate that ADAMs are important regulators of EGFR ligand shedding and EGFR activation in tumour cells, also contributing to the productive "cross-talk" between diverse signalling pathways and EGFR. Of particular interest is TACE/ADAM17, where Borrell-Pages et al. have now demonstrated a critical role in tumourigenesis, and EGFR signalling in breast cancer. While their individual signalling role and cellular impact remains to be defined, an increasing cohort of ADAMs are prominent in breast tumours. Borrell-Pages et al. also detected ADAM10 within normal and cancerous breast, while ADAM12 is overexpressed in several breast cancer cell lines [8] and ADAM9 is enriched in node positive disease [9]. Thus, given the prevalence and emerging signalling importance of ADAMs in breast cancer, ADAMmediated ligand shedding may provide interesting new avenues for therapeutic exploitation to abrogate EGFR signalling, particularly since it is now clear that EGFR inhibition has considerable potential to uncouple diverse mito- genic inputs. Such therapies may confer important advantages over existing anti-EGFR strategies, since ADAMmediated ectodomain shedding regulates many additional proteins linked to signal transduction and neoplastic cell behaviour. Thus, ADAMs can cleave cytokines, receptors (erbB4, MET, NGFR, PDGFR, TNFR, IL receptor), additional ligands (neuregulins), IGF binding proteins, and $\mathrm{NOTCH}$ signalling elements [10]. Future development of ADAM-selective inhibitors will be essential if unwanted side effects, previously noted with broad-spectrum metalloprotease inhibitors, are to be avoided.

\section{Competing interests}

JMW Gee has research grant funding from AstraZeneca.

\section{References}

1. Borrell-Pages M, Rojo F, Albanell J, Baselga J, Arribas J: TACE is required for the activation of the EGFR by TGF-alpha in tumors. EMBO J 2003, 22:1114-1124.

2. Gschwind A, Hart S, Fischer OM, Ullrich A: TACE cleavage of proamphiregulin regulates GPCR-induced proliferation and motility of cancer cells. EMBO J 2003, 22:2411-2421.

3. Yan Y, Shirakabe K, Werb Z: The metalloprotease Kuzbanian (ADAM10) mediates the transactivation of EGF receptor by $G$ protein-coupled receptors. J Cell Biol 2002, 158:21-26.

4. Dong J, Opresko LK, Dempsey PJ, Lauffenburger DA, Coffey RJ, Wiley HS: Metalloprotease-mediated ligand release regulates autocrine signalling through the epidermal growth factor receptor. Proc Natl Acad Sci USA 1999, 96:6235-6240.

5. Filardo EJ: Epidermal growth factor receptor (EGFR) transactivation by estrogen via the G-protein-coupled receptor, GPR30: a novel signalling pathway with potential significance for breast cancer. J Steroid Biochem Mol Biol 2002, 80:231-238.

6. Roudabush FL, Pierce KL, Maudsley S, Khan KD, Luttrell L M: Transactivation of the EGF receptor mediates IGF-1-stimulated shc phosphorylation and ERK1/2 activation in COS-7 cells. J Biol Chem 2002, 275:22583-22589.

7. Gilmore AP, Valentijn AJ, Wang $P$, Ranger AM, Bundred $N$, O'Hare MJ, Wakeling A, Korsmeyer SJ, Streuli CH: Activation of BAD by therapeutic inhibition of epidermal growth factor receptor and transactivation by insulin-like growth factor receptor. J Biol Chem 2002, 277:27643-27650.

8. Iba K, Albrechtsen R, Gilpin BJ, Loechel F, Wewer UM: Cysteinerich domain of human ADAM 12 (meltrin alpha) supports tumor cell adhesion. Am J Pathol 1999, 154:1489-1501.

9. O'Shea C, McKie N, Buggy Y, Duggan C, Hill AD, McDermott E, O'Higgins N, Duffy MJ: Expression of ADAM9 mRNA and protein in human breast cancer. Int J Cancer 2003, 105:754-761.

10. Mishra-Gorur K, Rand MD, Perez-Villamil B, Artavanis-Tsakonas S: Down-regulation of Delta by proteolytic processing. J Cell Biol 2002, 159:313-324.

\section{Note}

This article is based on papers highlighted by Faculty of 1000 (http://www.facultyof1000.com/start.asp), a web-based literature awareness service. Faculty of 1000 evaluations available for articles cited in this report may be viewed at: http://www.breast-cancer-research.com/reports/bcr637.asp

\section{Correspondence}

Julia MW Gee, Tenovus Centre for Cancer Research, Welsh School of Pharmacy, Cardiff University, Redwood Building, King Edward VII Avenue, Cardiff CF10 3XF, Wales, UK, Tel: +44 292087 5226; Fax: +44292087 5152; e-mail: gee@cardiff.ac.uk 